\title{
SCHEDULING AND SEPARATING DEPARTURES CROSSING ARRIVAL FLOWS IN SHARED AIRSPACE
}

\author{
Eric Chevalley, San Jose State University / NASA Ames Research Center, Moffett Field, CA \\ Bonny Parke, Paul Lee, Faisal Omar, Hwasoo Lee, Nancy Bienert, Joshua M. Kraut, San Jose State \\ University / NASA Ames Research Center, Moffett Field, CA \\ Everett Palmer, NASA Ames Research Center, Moffett Field, CA
}

\begin{abstract}
Flight efficiency and reduction of flight delays are among the primary goals of NextGen. In this paper, we propose a concept of shared airspace where departures fly across arrival flows, provided gaps are available in these flows. We have explored solutions to separate departures temporally from arrival traffic and pre-arranged procedures to support controllers' decisions. We conducted a Human-in-the-Loop simulation and assessed the efficiency and safety of 96 departures from the San Jose airport (SJC) climbing across the arrival airspace of the Oakland and San Francisco arrival flows. In our simulation, the SJC tower had a tool to schedule departures to fly across predicted gaps in the arrival flow. When departures were mistimed and separation could not be ensured, a safe but less efficient route was provided to the departures to fly under the arrival flows. A coordination using a point-out procedure allowed the arrival controller to control the SJC departures right after takeoff. We manipulated the accuracy of departure time (accurate vs. inaccurate) as well as which sector took control of the departures after takeoff (departure vs. arrival sector) in a $2 \times 2$ full factorial plan. Results show that coordination time decreased and climb efficiency increased when the arrival sector controlled the aircraft right after takeoff. Also, climb efficiency increased when the departure times were more accurate. Coordination was shown to be a critical component of tactical operations in shared airspace. Although workload, coordination, and safety were judged by controllers as acceptable in the simulation, it appears that in the field, controllers would need improved tools and coordination procedures to support this procedure.
\end{abstract}

\section{Introduction}

Today in terminal environments, arrival and departure flows are decoupled and assigned to distinct arrival and departure sectors. This spatial segregation avoids interactions and procedurally provides for separation between aircraft. This results in safe but inefficient routes in places where efficient routes would otherwise overlap.

In metroplex environments, efficiency and delays can be further compromised by the density and complexity of operations. Metroplex is defined by the Joint Planning and Development Office (JPDO) as an area with high traffic demand served by two or more airports with arrival and departure operations that are highly interdependent [1]. Metroplex interdependencies stem from different traffic flows sharing common fixes, paths or airspace volumes within the metroplex airspace. [2]. These interdependencies can be coordinated by either separating traffic across space or separating traffic across time. When traffic uses the same volume of airspace, it can be separated laterally, vertically, or temporally. When traffic uses the same lateral point, it can be separated vertically or temporally.

These types of separation are control strategies that have different costs and benefits. Spatial separation decouples traffic demand and relies less on the precision of when aircraft cross a given point. However, having distinct routes in segregated airspaces reduces airspace capacity. Routes may be longer and require altitude constraints to accommodate other routes and sectors, which also results in fuel and time inefficiencies. For controllers, the division of airspace spatially reduces traffic complexity. It clearly divides tasks and responsibilities between controllers. But this division has a cost when interdependencies exist between controllers. In current control facilities, Letters Of Agreement and Standard Operating Procedures regulate coordination needs between sectors.

Temporal separation involves dealing with multiple traffic demands and coordinating the use of shared resources, e.g., common fixes and runways. Today it is mostly used to space or merge traffic to a 
common destination or to intersecting runways. Temporal separation optimizes the use of airspace by accommodating multiple interdependent demands, but it also requires precision in timing. Temporal separation can be managed on a first-come firstserved basis, but it is not an efficient strategy to manage multiple conflicting demands or high traffic density. The use of shared resources can be exceeded by high demand and create choke points. Temporal separation can therefore benefit from scheduling technology [3, 4].

At NASA Ames Research Center, the Airspace System Project aims at developing scheduling and automation technologies for complex operational choke points in metroplex airspace. One of the objectives is to develop concepts and technologies to maximize performance for interacting arrival and departure operations. Recent modeling studies at NASA Ames have shown that the hybrid use of spatial and temporal separation supports more efficient routes in the metroplex environment $[3,4]$.

\section{Our Study}

In this study, we explore scheduling solutions to coordinate the demand of both arrival and departures over common waypoints in temporally shared airspace. We use the term "Sharing Of Airspace Resources" (SOAR) to describe the concept of efficient arrival and departure routes crossing each other and sharing a common airspace.

We chose the NorCal TRACON environment as a specific example of a generic problem.

\section{Shared Airspace}

The San Francisco Bay area is a metroplex environment with three large airports within $20 \mathrm{~nm}$ of each other. San Francisco International Airport (SFO) and Oakland International Airport (OAK) are on each side of the bay $10 \mathrm{~nm}$ apart from each other. San Jose International Airport (SJC) is on the south of the bay, $20 \mathrm{~nm}$ apart from SFO and OAK. Today, departures from San Jose to the northeast fly the LOUPE1 departure. Aircraft on this departure route fly a 360 degree turn over the city at 12,000 feet and then head to the north above arrival traffic coming into Oakland and San Francisco from the southwest. This highly inefficient route is designed to avoid the Oakland and San Francisco arrival flows, as shown in Figure 1.

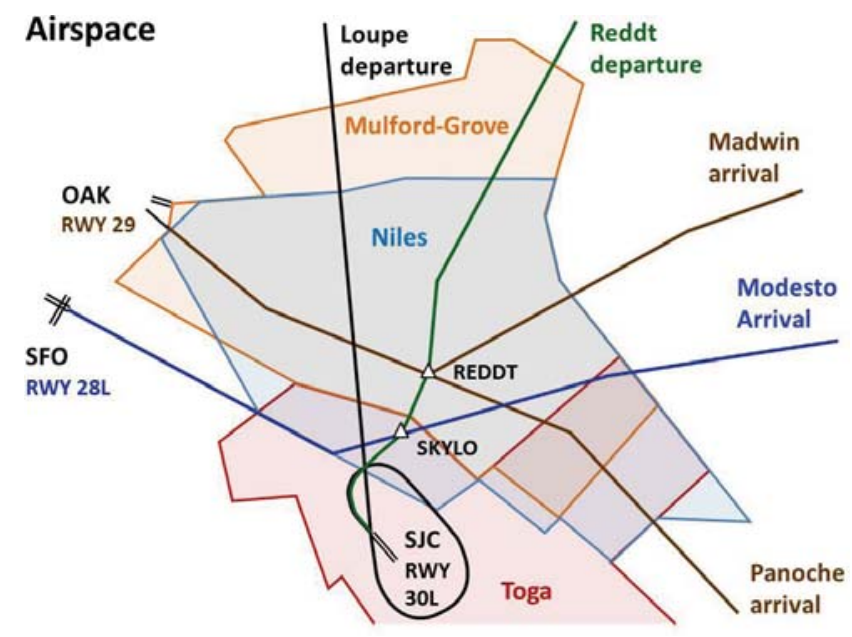

Figure 1. Routes and Sectors

We adapted existing Standard Instrument Departures (SID) and Standard Terminal Arrival Routes (STAR) to create a new departure from SJC that flies directly to the northeast, called REDDT1. The REDDT1 departure is derived from the Sunol6 departure for turboprops. The new REDDT1 route crosses the airspace of both arrival routes. The standard procedure is to fly this route safely under both arrival flows at 5,000 feet, i.e. to cross both the SKYLO and REDDT waypoints at 5,000ft. On the arrival routes, traffic is expected to cross SKYLO at $7,000 \mathrm{ft}$ and REDDT at 6,000ft. The altitude restrictions at SKYLO and REDDT allow departures to fly the route safely in case of a loss of radio communication.

Currently, the Madwin and Panoche STAR merge at the SUNOL waypoint. This waypoint is in class $\mathrm{C}$ airspace. Because the REDDT1 departures are flown only by jets, we moved that waypoint to the west into class B airspace and renamed it REDDT. Moving that point also created a better angle between the Modesto and REDDT1 routes to allow divergence between traffic flows.

The REDDT1 departure provides two advantages over the LOUPE1 departure. First, it is a shorter route. Second, the altitude restrictions at SKYLO and REDDT can be lifted provided there is an available gap in the arrival flows. Combined with a scheduling tool, the departures could be temporally separated from the arrivals and climb more efficiently. This was the main operational goal of the study. 
We did not create a sector around the Reddt 1 departure since the REDDT1 departure flies across the standard arrival sectors until it reaches $12,000 \mathrm{ft}$ or $24 \mathrm{~nm}$ out, as shown in Figures 1 and 2. The REDDT1 first flies in the Mulford-Grove combined arrival sector. If the aircraft is level at SKYLO and REDDT, it will stay in the Mulford-Grove sector. If the altitude restriction is lifted before Reddt, the aircraft will climb and eventually enter the Niles arrival sector. Figure 2 shows the optimal REDDT1 departure climb profile we used for B737-800 aircraft. It shows how the three different climb profiles of the route penetrate the arrival sectors. The black line shows the safe route where aircraft fly under the arrival routes and stay in the MulfordGrove sector. The red line is the route of aircraft leveling at SKYLO and then climbing at or above REDDT, eventually entering the Niles sector. The blue line is the route of aircraft climbing continuously at or above both SKYLO and REDDT, also eventually entering the Niles sector. The departing REDDT1 B738 aircraft reach 5,000ft approximately $3 \mathrm{~nm}$ before SKYLO, $4 \mathrm{~nm}$ before leaving the Toga sector. The triangles indicate the expected altitudes of the departures (in white), the Modesto arrivals (in blue) and the Oakland arrivals (in brown).

As can be seen, the REDDT1 departures climb across one or two arrival sectors for a brief period of time before entering a departure sector again. This creates a need to coordinate control of the aircraft, especially since they may no longer be spatially separated from the Oakland arrivals, which creates a need to separate the departures temporally from the arrivals.

These needs for coordination are created by the interdependencies and uncertainties created in this shared airspace. Different traffic flows share the same airspace and cross the same fix. Also, the REDDT1 departures may cross the shared fix at different time and altitude.

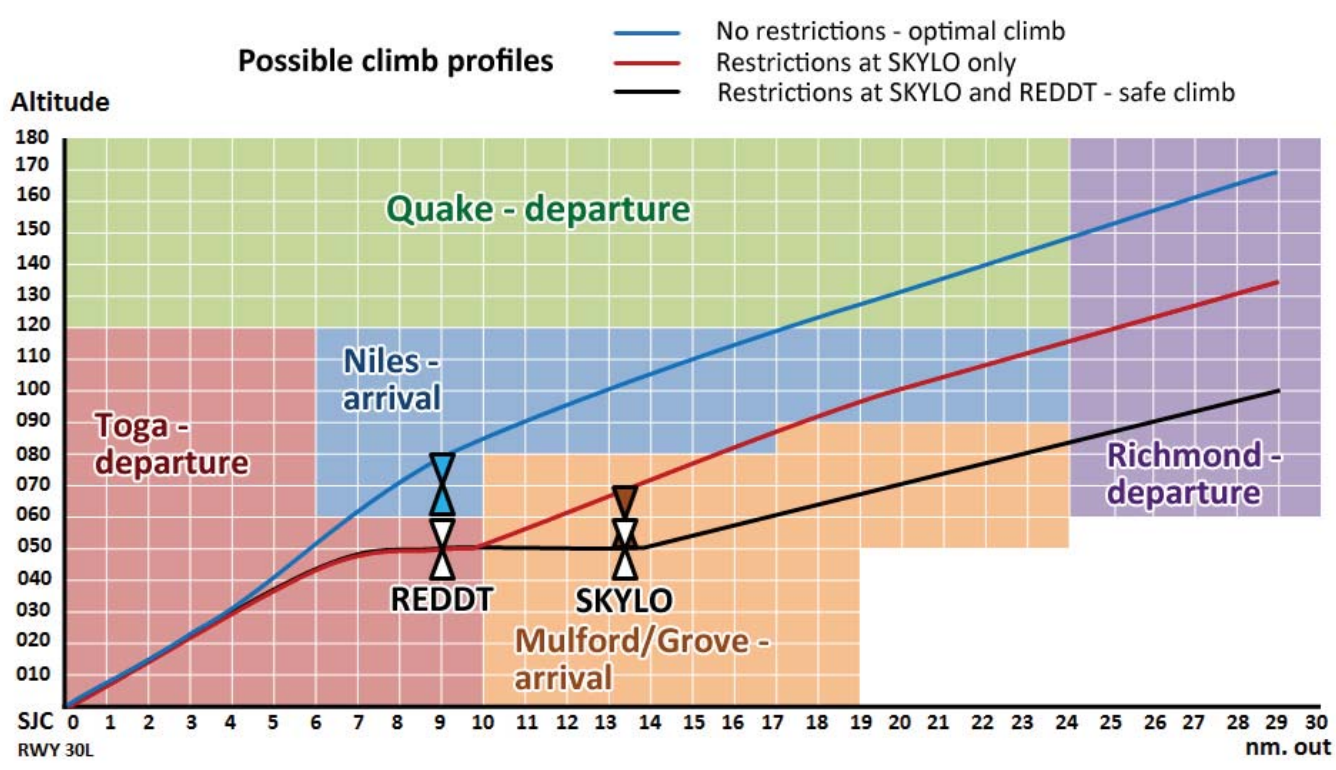

Figure 2. Possible Climb Profiles Across Sectors

\section{Coordination}

The need to coordinate leads to two main questions. First, who makes the decision to climb the departures and thus who is responsible for separating the REDDT1 departures from the arrivals? Second, how can the SJC tower schedule REDDT1 departures to fly through gaps in the arrival flows in a timely manner? To answer these questions, we designed a coordination procedure to support the control of the departures, and we developed a scheduling tool to support the timing of departures from the tower.

\section{Coordination Procedures between Sectors}

The Mulford-Grove controller (henceforth called Mulford) decided whether to climb REDDT1 departures to a higher altitude, and thus was 
responsible for separating them from arrivals. However, the Niles controller could veto the decision for an aircraft to enter Niles' sector. This coordination was done by point-out and was specified in a Standard Operating Procedure.

Another problem was the limited time for Mulford to decide whether to climb departures. The departing REDDT1 departures would be on Toga's SJC departure frequency until shortly before entering Mulford airspace at which time the aircraft would be at altitude level. So we developed another Standard Operating Procedure to coordinate between Mulford and Toga, giving an earlier control to Mulford.

Both Standard Operating Procedures are further detailed in the Method sections.

\section{Temporal Coordination: Scheduling Arrivals and Crossing Departures}

Scheduling is the most efficient way to allocate the use of the same resource and thus reduce uncertainties in temporal demand. In our concept, both arrivals and departures were scheduled to cross REDDT, the common fix. Assumptions about arrivals and departures were different. Arrivals flows have the most reliable time predictability. We used that as a known parameter to schedule departures. The arrival schedule allowed the creation of predictable gaps that would allow for unreliable departure times. We used the Controller Managed Spacing (CMS) tools developed in the Airspace Operation Laboratory [5] These tools work with a scheduler that gives a precise time for aircraft to meet at the runway threshold (Scheduled Time of Arrival, STA). The scheduler computes time from the meter fix at the boundary of the TRACON down to the runway. The main tool in the suite of CMS tools is the "slot marker." Slot markers are circles that represent where aircraft should be along its nominal route to meet its STA. When an aircraft is inside its slot marker, it is on time. Controllers can vector aircraft and use slot markers as a target. (CMS tools also include speed advisories and Early/Late indicators, which were not included in this simulation.) CMS tools are based on nominal routes, with expected altitudes and speeds on the procedures, and adjust for forecast winds.

In our study, the STAs of arrival aircraft were frozen on the schedule to the runways, meaning that the arrivals were committed to a STA. Controllers were instructed to keep arrival traffic on their route and in their slot marker. The departure times, on the other hand, were not frozen but floating to allow for flexibility. Departures did not use slot markers.

We adapted the Departure Flow Management (DFM) system to schedule departures and to coordinate the crossing times with arrivals. DFM allows an airport tower to schedule aircraft in available time slots [6]. The time slots reflect available times at a departure meter fix. Departure meter fixes are used to control flows into adjacent centers towards major destinations. In our study, the tower scheduled a REDDT1 departure based on the location of an aircraft on the runway departure timeline that matched a gap in the arrival flow. Gaps are excess spacing at the crossing fix propagated back onto the runway departure timeline, using a nominal flying time from the runway to the crossing fix. Gaps are comparable to time slots. Both gaps and time slots support the allocation and distribution of aircraft across time and space. However, gaps are reflective of the relative spacing between two arrivals and can be large at times. In our concept, gaps are controlled by the TRACON Traffic Management Unit. The TMU can decide to remove gaps or can set different buffer times between the gaps and the leading and trailing arrivals. The buffers provide a minimum separation with the leading or trailing aircraft with any aircraft inside the gap. We used 90 seconds of buffer on the front and the back of gaps to allow for $3 \mathrm{~nm}$ lateral separation. In the following example of runway timeline (Figure 3), gaps are indicated in dark blue. Callsigns are color-coded by departure routes: yellow for the LOUPE1, turquoise for the SanJose9, and magenta for the Reddt1. A Reddt 1 departure scheduled to depart to meet a gap is expected to cross REDDT inside the actual gap between arrivals on the Oakland arrival flow.

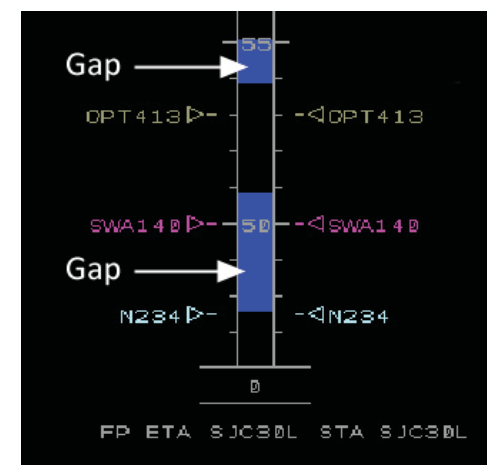

Figure 3. Timeline Display at SJC Tower 
Precise departures times are more difficult to predict than arrival times. Today, Call For Release (CFR) procedures give departures a $-1 \mathrm{~min}+2 \mathrm{~min}$ departure time window. In addition, not all departures meet that time window. Such uncertainty leads to aircraft missing slots, increased delays and added workload for controllers $[7,8]$.

Our scheduling concept allows for some flexibility in the departure time. For the tower, the gaps are not specific time slots, but rather a window of time when the tower can schedule departures depending on what is best for surface operations. Departures can be scheduled as soon as arrivals enter the TRACON and the STAs are known. This can be up to 20 minutes prior to departure. The DFM system eliminates the need for the tower to coordinate with the TRACON for a release time over the phone. To avoid unwanted departure times, the TRACON could require a conditional use of the gaps. This concept leverages the predictability of the arrival times and accommodates the uncertainty of departures times. It also minimizes the coordination process to releasing departures.

\section{Method}

We tested our concept in a high fidelity Humanin-the-Loop simulation at the Airspace Operation Laboratory (AOL) using the Multi Aircraft Control System (MACS) software [9].

\section{Experiment Design}

The simulation was a $2 \times 2 \times 2$ full factorial design. Each of the following were fully crossed: First Sector (Toga vs. Mulford), Tool Reliability (Reliable vs. Unreliable), and Gap (REDDT vs. SKYLO).

\section{First Sector}

In half of the runs, the first sector to control the REDDT1 departures was either Toga the departure sector or Mulford the next arrival sector. When Toga was the first sector, SJC Tower would request pilots to contact Toga's frequency, who would then later transfer communication to Mulford. When Mulford was first, SJC Tower would request pilots to contact Mulford's frequency directly. The consequence for Mulford was that it had control of the aircraft a few miles off its boundary or on contact just after the aircraft had taken off. We hypothesized that Mulford would have more time to coordinate with Niles and make a decision to climb when Mulford was the first sector, and climb performances would improve.

\section{Tool Reliability/Departure Accuracy}

In half of the runs, all the departures took off inside the predicted gap on the scheduler (Reliable Condition). In the other half of the runs, half of the departures took off outside of the predicted gap, and the other half inside the predicted gap (Unreliable Condition). The rationale for this manipulation was to test the impact of the precision of the tool on the controllers' decisions to climb safely above the nominal route. In each run, 8 departures were scheduled by the tower confederate. There were 4 positions of the departure time relative to the gap: $60 \mathrm{sec}$ and $30 \mathrm{sec}$ inside a gap, or $60 \mathrm{sec}$ and $30 \mathrm{sec}$ outside of the gap, as shown in Figure 4. These positions were also relative to the front and to the back of a gap. This yielded a total of 8 different possibilities. The order of the 8 positions was counterbalanced so that each run had a different order and an equal distribution of each position for each run.

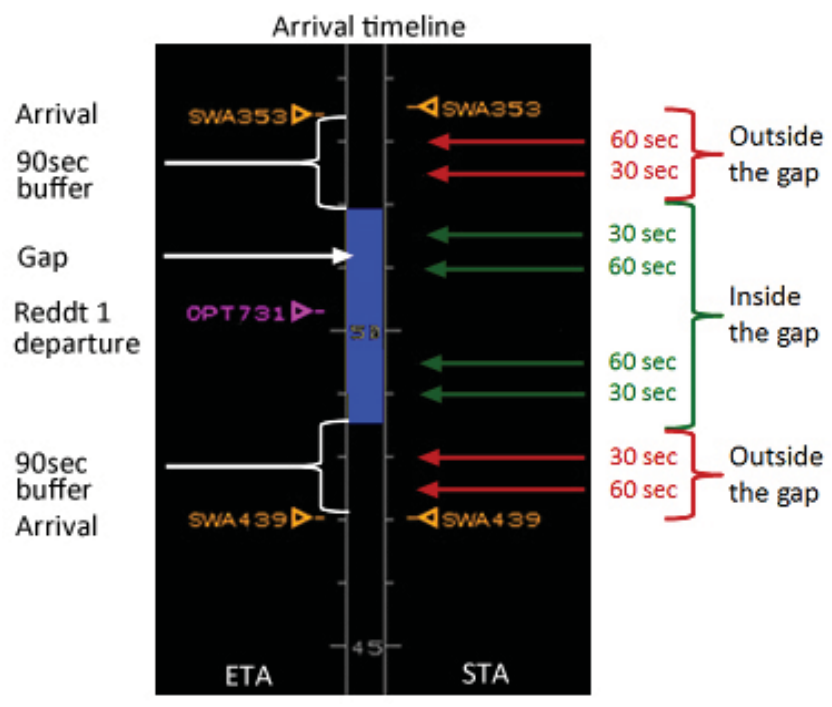

\section{Figure 4. Departure Accuracy Conditions}

The four relative positions of the departures provided additional opportunities for our analyses. They could be treated as more or less accurate departures (Departure Accuracy). In addition to studying the impact of tool precision then, is studying the impact of the actual position of the departure on the controllers' decisions. We hypothesized that aircraft departing outside of the predicted gaps would 
be kept at level altitude until clear of arrival traffic more often than aircraft predicted to fly through gaps.

\section{Gaps at REDDT and SKYLO}

It became apparent in the first few runs that controllers were vectoring departures to separate them from arrival traffic at REDDT, but also at SKYLO. Controllers could then climb aircraft above the Modesto arrival flow, instead of keeping aircraft underneath it. REDDT1 departures then only occasionally crossed the REDDT waypoint where the gaps were. After repeating our 4 main conditions 2 times (8 runs) with gaps at REDDT, we ran the 4 conditions again with gaps at SKYLO (4 runs).

The 12 runs with the associated experimental factors are listed in Table 1 below.

Table 1. Experimental Design

\begin{tabular}{llll} 
Run \# & Gap at & First Sector & Tool reliability \\
\hline 1 & REDDT & Toga & Reliable \\
2 & REDDT & Toga & Unreliable \\
3 & REDDT & Mulford & Unreliable \\
4 & REDDT & Toga & Reliable \\
5 & REDDT & Mulford & Unreliable \\
6 & REDDT & Mulford & Reliable \\
7 & REDDT & Mulford & Reliable \\
8 & REDDT & Toga & Unreliable \\
9 & SKYLO & Mulford & Reliable \\
10 & SKYLO & Toga & Reliable \\
11 & SKYLO & Mulford & Unreliable \\
12 & SKYLO & Toga & Unreliable
\end{tabular}

\section{Participants}

Five retired controllers with experience in TRACON airspace staffed five sectors. The two most recently retired controllers rotated through the Niles and Mulford positions. A third controller staffed Toga, the SJC departure sector. A fourth staffed Sunol-Cedar, the SFO and OAK arrival combined feeder sectors. A fifth staffed Final, the final approach sector to SFO (called "Foster" at NCT). One researcher was a confederate and acted as a local controller at San Jose Tower. Six pseudo-pilots participated. Each pseudo-pilot was responsible for flying aircraft in a sector. All pilots were students from the Aviation department at San Jose State University.

\section{Traffic Scenario}

We developed two traffic scenarios derived from actual traffic data. Both had similar attributes. Arrival traffic was for SFO and OAK and departures from SJC. Some overflight and Visual Flight Rules (VFR) traffic were included for realism. The Modesto arrival traffic rate met the maximum landing capacity for the SFO runway 28R. Both Oakland arrivals were populated with more traffic than today. Gaps occurred when excess spacing existed between arrivals. This occurred naturally in the Modesto flow. Large gaps occurred when Golden6 arrivals were scheduled to merge with the Modesto flow downstream of SKYLO. Large gaps were built into the Oakland arrival flow to allow for REDDT1 departures to climb. Departure aircraft from SJC were scheduled to either depart on the LOUPE1 departure or the SanJose9 departure. There were 12 departures per run and 12 runs total.

\section{Apparatus}

Standard Terminal Automation Replacement System (STARS) screen displays were emulated on the MACS software. MACS provides a high fidelity environment to prototype the scheduling tools, to simulate the air traffic and to collect data [10].

Two tools assisted controllers: J-rings and timelines. J-rings are circles around aircraft that can be set at any size. In this study the size was set to the minimum separation $(3 \mathrm{~nm})$.

Timelines depicted two relative positions of aircraft to a specific waypoint. One position showed an Estimated Time of Arrival (ETA) and the other showed an STA of aircraft at the waypoint. MACS computes aircraft trajectories to determine ETAs, based on flight plans, altitude and speed constraints, and forecast winds. MACS then uses ground-based trajectory and scheduling criteria, such spacing values, to compute the STA. Slot markers follow the STA. An STA shows when aircraft should cross the waypoint. An ETA is when it is predicted to actually cross.

There were different configurations of timelines: two timelines for the arrivals to OAK and SFO runways (OAK29 and SFO28R), and another timeline for the departure from SJC30L. In the case of the departure timeline, the time to the runway represented the time to takeoff. There was also a 
timeline to the crossing fix which depicted gaps and the departures' ETA and arrivals' ETA and STA to the cross point.

\section{Operational Procedure}

We developed operational procedures to schedules departures and for coordinating control of the departures between the arrival sectors.

\section{Scheduling Departures}

In each scenario, departures out of SJC were filed to fly either the LOUPE1 departure to the northeast or the SJC9 departure to the south. The tower confederate acted as a cab coordinator who would coordinate with both the Clearance Delivery and the Local controller positions. The tower confederate looked at the departure timeline and checked which flight departure time would be likely to match a gap.

We assumed that pilots would accept an amendment to the standard LOUPE1 departure to a REDDT1 departure 10-15 minutes prior to take off because the REDDT1 is a shorter route. At SJC, the aircraft is likely to be at the gate at this time. At a busy airport, pilots can expect to amend their departure even while waiting near the runway. We assumed the pilots would load a new departure in their Flight Management System (FMS).

Once the pilots accepted the amendment, the controller inserted it in the ground system and assigned a new departure time, if that was necessary. We assumed that pilots would accept a few minutes of departure time delay since the flight time of REDDT1 departures is about 6 minutes shorter than that of the LOUPE1 departures.

For each run, the tower confederate scheduled aircraft either inside or outside the gap according to which Departure Accuracy conditions they were in. We counter balanced both scenarios and the Departure Accuracy conditions, thus providing a unique combination for each departure.

\section{Controllers' Coordination: Point-out and Handoff}

Once the departure took off, the tower confederate requested that the pilot contact the first sector, Toga or Mulford, depending on the experimental condition. The request took place when aircraft was between $500 \mathrm{ft}$ and $1,000 \mathrm{ft}$ off the ground.

When radio contact was established with Toga first, Toga would immediately hand-off the datablock to Mulford. However, in this condition, the Standard Operating Procedure required Toga to wait until the REDDT1 aircraft reached 2000ft and was $1.8 \mathrm{DME}$ from the SJC VOR to request that the pilots contact Mulford's frequency. When radio contact was established with Mulford first, the Standard Operating Procedure allowed Mulford to have control of that aircraft on contact and to display its datablock to Toga. Any lateral deviation from the routes required further coordination with Toga.

Once Mulford had control of the aircraft, s/he had to decide whether the REDDT1 departure altitude restriction could be lifted to allow the aircraft to climb through Niles airspace. If traffic appeared to permit this, Mulford pointed out the aircraft to Niles. If Niles approved the point-out, Mulford could clear the aircraft to 11,000 feet, the ceiling altitude of Niles sector, and could hand-off the datablock to Quake, a departure sector. If Niles or Mulford needed to vector the departure aircraft, verbal coordination was required. This point-out procedure was specified in a Standard Operating Procedure and read as follows: "Mulford has control for climb with a point out with Niles. A point out approval authorizes Mulford to climb departures through Niles' airspace. Lateral deviations from the route require further coordination." Mulford also displayed the REDDT1 departure to the Foster sector, so that Foster would not descend any arrival aircraft until past SKYLO. When lifting the altitude restrictions was not possible, Mulford did not point out to Niles and kept the aircraft at 5000 feet in Mulford's airspace.

The recommended procedure was for Mulford to keep aircraft under the Modesto arrival flow, and then decide whether to climb REDDT1 departure aircraft based on the gaps in the Oakland arrival flow.

Controller positions were dispersed across two control rooms. We allocated the positions in such a way that a neighboring airspace sector would be in a separate room. For instance, Mulford was separated from Toga and Niles. This was done to force controllers to use the point out tool and the voice communication system, and avoid face-to-face coordination. This also reflects the actual allocation 
of the sectors across different control areas at Northern California TRACON.

\section{Experimental Procedure}

We tested our concept and MACS emulation with the participants several times prior to the study. The study itself took place over four days. On the first day, we briefed controllers about their tasks and responsibilities, and particularly about the operational procedures. Then controllers trained during four practice runs with our four main conditions using separate scenarios from those used in the actual data collection runs. The following three days, controllers participated in twelve runs for data collection. Finally, a debrief discussion with controllers and pseudo pilots concluded the study.

Controllers answered questions after each run online and at the end of the study on paper. The questions pertained to workload, acceptability, feasibility and safety of the operation and coordination.

\section{Results}

Twelve runs produced 96 departures flying in arrival airspace, 8 departures per run. Each departure was treated as a single case since its departure time and therefore its position relative to other aircraft was never the same.

We analyzed data for coordination effort, climb efficiency, safety, and acceptability. We used time in seconds to measure the timing of action events, such as when point-outs or handoffs started and ended, and when the pilots were cleared to climb to 11,000 feet. We also used altitude to measure climb performance, as well as nautical miles to assess the loss of lateral separation.

We begin by presenting analyses based on our original design: First Sector X Tool Reliability and First Sector X Departure Accuracy for the first 8 runs $(N=64)$ when the gaps were at REDDT. Then, we present analyses for runs with gaps at SKYLO $(N=$ 32 ) and finally, we present results comparing scheduling to gaps at REDDT and SKYLO.

\section{Coordination: First Sector and Point-Out}

Early coordination allowed controller to make decisions about the Reddt departures earlier across all runs. In the first 8 runs, when gaps were situated at
REDDT, we found significant main effects of First Sector on the timing of point outs, as shown in Table 2 below. Point outs started an average of 20 seconds earlier when the first sector to control departures was Mulford $(M=27.6 \mathrm{sec}, S D=18.2)$ than Toga $(M=$ $47.6 \mathrm{sec}, S D=22.4), F(1,60)=14.9, p<.001)$. Table 2 lists the mean time (Standard Deviations) in seconds from takeoff and aircraft altitude in feet when cleared to climb to 11000 feet as a function of First Sector.

We also tested whether early coordination had an effect on the altitude aircraft were at when cleared to climb to 11000 feet. First Sector had a marginal main effect. Aircraft was at a lower altitude when Mulford was first $(M=4,184 \mathrm{ft}, S D=1,250)$, than when Toga was $(M=4,632 \mathrm{ft}$, SD 737), $F(1,60)=3.0$, $p=.09$ (ns) as shown in Table 2. This marginal effect would become significant ( $p=.045$, one-tail) if we initially assumed a unilateral directional effect of First Sector (Mulford over Toga). Time wise, aircraft seemed to be cleared to climb to 11,000 feet slightly earlier when Mulford was first $(M=138.0 \mathrm{sec}, S D=$ 46.3) compared to Toga $(M=151.7 \mathrm{sec}, S D=35.3)$. However no significant main effect was found, $F(1,60)=1.8, p=.18(\mathrm{~ns})$.

Table 2. Point-Out and Aircraft Altitude

\begin{tabular}{|c|c|c|c|}
\hline \multicolumn{4}{|c|}{ First Sector } \\
\hline & $\begin{array}{r}\text { Mulford } \\
M(S D) \\
\end{array}$ & $\begin{array}{r}\text { Toga } \\
M(S D) \\
\end{array}$ & $F$ \\
\hline \multicolumn{4}{|l|}{ Gap at REDDT } \\
\hline Point-out start time & $\begin{array}{r}27.6 \\
(18.2)\end{array}$ & $\begin{array}{r}47.6 \\
(22.4)\end{array}$ & $14.9 * *$ \\
\hline $\begin{array}{l}\text { Aircraft altitude when } \\
\text { cleared to } 11,000 \text { feet }\end{array}$ & $\begin{array}{r}4,184 \\
(1250) \\
\end{array}$ & $\begin{array}{l}4,632 \\
(737) \\
\end{array}$ & $3.0^{\mathrm{a}}$ \\
\hline \multicolumn{4}{|l|}{ Gap at SKYLO } \\
\hline Point-out start time & $\begin{array}{r}24.0 \\
(19.0)\end{array}$ & $\begin{array}{r}38.6 \\
(18.8)\end{array}$ & $4.8^{*}$ \\
\hline $\begin{array}{l}\text { Aircraft altitude when } \\
\text { cleared to } 11,000 \text { feet }\end{array}$ & $\begin{array}{r}3,450 \\
(1,379) \\
\end{array}$ & $\begin{array}{l}4,187 \\
(995) \\
\end{array}$ & $3.0^{\mathrm{a}}$ \\
\hline \multicolumn{4}{|l|}{ All runs $(1-12)$} \\
\hline Point-out start time & $\begin{array}{r}26.4 \\
(18.4)\end{array}$ & $\begin{array}{r}44.6 \\
(21.5)\end{array}$ & $19.9^{* *}$ \\
\hline $\begin{array}{l}\text { Aircraft altitude when } \\
\text { cleared to } 11,000 \text { feet }\end{array}$ & $\begin{array}{r}3,939 \\
(1,326) \\
\end{array}$ & $\begin{array}{l}4,484 \\
(848)\end{array}$ & $5.8^{*}$ \\
\hline
\end{tabular}


feet, as well as with the altitude of the aircraft at the time of the clearance. The later that point outs started, the later that clearances were given to 11,000 feet $(r$ $(64)=.29, p<.05)$ and the higher altitude of the aircraft $(r(64)=.28, p<.05)$.

In the last four runs, when gaps were at SKYLO, the results were similar. Point outs started earlier when the first sector was Mulford $(M=24 \mathrm{sec}, S D=$ 19) instead of Toga $(M=38.6 \mathrm{sec}, S D=18.8)$, $F(1,30)=4.8, p=<.05$, as shown in Table 2. Also, First Sector had a marginal effect on when aircraft were cleared to climb to $11,000 \mathrm{ft}$. Aircraft were at a lower altitude when the first sector was Mulford $(M=$ $3,450 \mathrm{ft}, S D=1,379)$, compared to Toga $(M=4,187$ $\mathrm{ft}, S D=995), F(1,30)=3.0, p=.09(\mathrm{~ns})$. This marginal effect would also become significant $(p=$ .045 ) if we assumed a unilateral directional effect of First Sector (Mulford over Toga).

When we considered the 12 runs together, the First Sector had a significant main effect on when aircraft were cleared to 11,000 feet, $F(1,94)=5.8, p$ $=.02$, as shown in Table 2. Departures were at lower altitude when Mulford was first $(M=3939 \mathrm{ft}, S D=$ 1326) compared to Toga $(M=4484 \mathrm{ft}, S D=848)$. Also, point outs started earlier when the first sector was Mulford $(M=26.4 \mathrm{sec}, S D=18.4)$ instead of Toga $(M=44.6 \mathrm{sec}, S D=21.5 ; F(1,94)=19.9, p=$ $.000)$. These results indicate that the aircraft climb performance benefitted from arrival controllers having control of the aircraft immediately after takeoff.

As expected, the First Sector condition was independent of the Tool Reliability/Departure Accuracy condition. Surprisingly, we did not find any Tool Reliability/Departure Accuracy effect on the altitude or the time aircraft were cleared to climb to 11,000 feet for the first 8 runs, when the gaps were at REDDT. Two explanations are possible. First, controllers tactically tried to climb departures through gaps in the Modesto traffic at SKYLO. If successful, this helped to achieve a vertical separation with arrival traffic at REDDT, making traffic at REDDT no longer a constraint. Second, controllers vectored a large proportion (44\%) of departures a few nautical miles to the left of the REDDT waypoint where they could fly above the arrival traffic, making these aircraft independent of arrival traffic and any gaps at REDDT. We describe this further in the decision analysis below. We then present significant differences between the runs with gaps at REDDT versus gaps at SKYLO.

\section{Controllers' Decision Analysis}

Figure 5 depicts the decisions controllers made for the REDDT1 departures when the gaps were at REDDT (left) for the first 8 runs, and at SKYLO (right) for the last 4 runs. Working from the left of Figure 5, the Mulford controller's first decision was either to leave a REDDT1 departure on its route or to vector it. In either case, the second decision involved looking for natural gaps in the SKYLO flow and deciding whether to keep the aircraft level or to climb it at or before SKYLO. This process was repeated for possible gaps in traffic at REDDT.

In the first 8 runs (depicted on the left of Figure 5), controllers climbed $100 \%$ (64) of the aircraft before REDDT. They vectored 44\% (28/64) of aircraft before SKYLO. Of the vectored aircraft, $46 \%$ $(13 / 28)$ stayed level until they passed SKYLO and then climbed before crossing the Oakland arrivals (west of REDDT). Of the 56\% (36/64) of aircraft staying on their route, $44 \%(16 / 36)$ climbed before SKYLO, and continued to climb at REDDT. The remaining 20 aircraft leveled at SKYLO, and then continued to climb before REDDT. Of all the departures with the gap at REDDT, 48\% (31/64) climbed before SKYLO and continued to climb before REDDT. The remaining 51\% (33/64) leveled before SKYLO, and then climbed before REDDT.

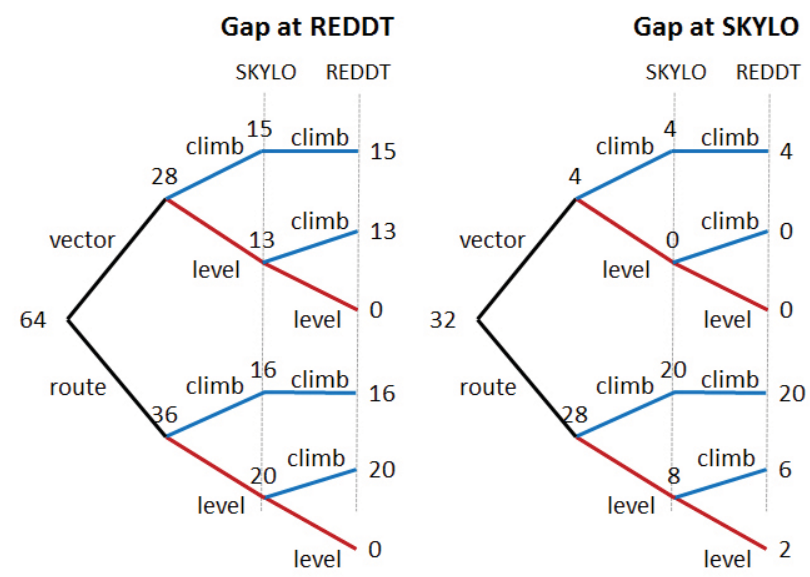

Figure 5. Decision Tree

In the last 4 runs, only $13 \%(4 / 32)$ of aircraft were vectored. These 4 aircraft were climbed before SKYLO and continued to climb before crossing the Oakland arrivals (west of REDDT). Of the remaining 
$87 \%(28 / 32)$ aircraft that stayed on their route, $71 \%$ $(20 / 32)$ of aircraft were climbed before SKYLO, and continue to climb before REDDT. The remaining 8 aircraft were leveled at SKYLO. Of those 8 aircraft, 6 were then climbed before REDDT. Only 2 aircraft were kept level at SKYLO and REDDT.

Controllers gradually favored vectoring departures over keeping them on their route during the first 8 runs $\left(\chi^{2}(7, N=64)=18.3, \mathrm{p}=.011\right)$. A Somers' $d$ test showed vectoring was dependent on the order of the runs $(t(7, N=64)=.397, \mathrm{p}=.004, d$ $=.397)$. In Run $1,25 \%$ of the aircraft were vectored, in Run 6,7 and $8,75 \%$ of the aircraft were vectored. In the last 4 runs (9-12), controllers kept all but 4 aircraft on the route. According to the controllers, it was simpler to leave an aircraft on its route if it was scheduled to fit in the gap on the Modesto flow. This was not possible in the first 8 runs.

\section{Climb Profiles}

Overall, in the first 8 runs, controllers climbed aircraft before REDDT, whether they leveled aircraft at 5,000 feet under the Modesto flow, or not. Table 3 shows the average altitude of aircraft for each decision. Aircraft that leveled under the Modesto flow and climbed after that crossed the Oakland flow on average at 8,748 feet. The arrival traffic crossed REDDT at 6,000 feet. The departures were well above the arrival traffic by the time they crossed the Oakland arrival flow ${ }^{1}$.

Controllers climbed aircraft before REDDT regardless of whether the aircraft was scheduled to fly inside a gap or not. Departure Accuracy did not have a main effect on the altitude of aircraft when it crossed REDDT or the arrival route $(60 \mathrm{sec}$ in $(M=$ $9,567 \mathrm{ft}), 30 \mathrm{sec}$ in $(M=9,946 \mathrm{ft}), 30 \mathrm{sec}$ out $(M=$ $10,113 \mathrm{ft}), 60 \mathrm{sec}$ out $(M=9,888 \mathrm{ft}), F(3,60)=0.4, p=$ $\mathrm{ns})$. The result was similar for aircraft that stayed on the route $(N=36)$. Also climbing or leveling at SKYLO did have a significant effect on the altitude crossing REDDT. Aircraft crossed REDDT at a higher altitude when they were already climbing at SKYLO $(M=10,850 \mathrm{ft}, S D=555)$, than when they were leveling at SKYLO $(M=8210 \mathrm{ft}, S D=910)$, $F(1,34)=103.5, p=.000$. This result suggests that leveling at SKYLO allowed departures to top arrival traffic at REDDT with enough vertical clearance. All

\footnotetext{
${ }^{1}$ The climb rate used for B738 in our simulation was optimal.
}

aircraft ended up climbing well above the Oakland traffic at REDDT.

Table 3. Altitude at Crossing Fixes

\begin{tabular}{lllll}
\hline \multicolumn{2}{l}{ Gap at REDDT } & & & \\
Decisions & & SKYLO & REDDT \\
\hline vector & climb & climb & 6726 & 11066 \\
vector & level & climb & 5008 & 9577 \\
vector & level & level & N/A & N/A \\
route & climb & climb & 7712 & 10850 \\
route & level & climb & 5010 & 8210 \\
route & level & level & N/A & N/A \\
& & & & \\
\hline Gap at SKYLO & & & \\
Decisions & & SKYLO & REDDT \\
\hline vector & climb & climb & 6780 & 11000 \\
vector & level & climb &. &. \\
vector & level & level &. &. \\
route & climb & climb & 8432 & 11036 \\
route & level & climb & 5000 & 7867 \\
route & level & level & 5000 & 5000
\end{tabular}

Controllers climbed aircraft before REDDT regardless of whether the aircraft was scheduled to fly inside a gap or not. Departure Accuracy did not have a main effect on the altitude of aircraft when it crossed REDDT or the arrival route (60sec in $(M=$ $9,567 \mathrm{ft}), 30 \mathrm{sec}$ in $(M=9,946 \mathrm{ft}), 30 \mathrm{sec}$ out $(M=$ $10,113 \mathrm{ft}), 60 \mathrm{sec}$ out $(M=9,888 \mathrm{ft}), F(3,60)=0.4, p=$ ns). The result was similar for aircraft that stayed on the route $(N=36)$. Also climbing or leveling at SKYLO did have a significant effect on the altitude crossing REDDT. Aircraft crossed REDDT at a higher altitude when they were already climbing at SKYLO $(M=10,850 \mathrm{ft}, S D=555)$, than when they were leveling at SKYLO $(M=8210 \mathrm{ft}, S D=910)$, $F(1,34)=103.5, p=.000$. This result suggests that leveling at SKYLO allowed departures to top arrival traffic at REDDT with enough vertical clearance. All aircraft ended up climbing well above the Oakland traffic at REDDT.

In comparison, Departure Accuracy significantly influenced the aircraft altitude at SKYLO when departures were planned to cross gaps there. Table 4 shows the means of altitude and Standard Deviations of aircraft crossing SKYLO as a function of Departure Accuracy. The breakdown of the 4 accuracy conditions indicates that the relative 
position influences how early the aircraft is climbed. This suggests controllers could not climb aircraft before SKYLO due to a lack of lateral separation. There is a main effect of departure accuracy on the altitude of aircraft crossing SKYLO, $F(3,23)=14.3$, $p=.000$. The more inside the gap the departure, the higher the altitude the aircraft crosses SKYLO. A departure scheduled 60 seconds outside a gap was only 30 seconds away from an arrival aircraft. In this case, aircraft were leveled at 5000 feet to maintain vertical separation. In contrast, aircraft scheduled 30 seconds or 60 seconds inside the gaps were, respectively, 120 and 150 seconds away from arrival aircraft, and thus could be climbed. Departures inside the gaps show the highest altitudes $(8,009 \mathrm{ft}$ vs. $8,850 \mathrm{ft}$, as shown in Table 4).

Table 4. Altitude at SKYLO by Accuracy

\begin{tabular}{lrr} 
& Altitude at SKYLO \\
\hline & M & SD \\
\hline Scheduled & & \\
60sec inside gap & 8850 & 926 \\
30sec inside gap & 8009 & 1624 \\
30sec outside gap & 5325 & 395 \\
60sec outside gap & 5000 & 0 \\
\hline
\end{tabular}

The aircraft altitudes at SKYLO result from the main effect of departure accuracy on the time and the altitude of when the aircraft was cleared to climb to 11,000 feet (see Figure $6 \& 7$ ).

We compared the Departure Accuracy condition on the gaps at REDDT and SKYLO. We found a significant interaction effect on the time and altitude of the aircraft when it is cleared to 11,000 feet (See Figure 6 and 7). For comparison purpose, we used aircraft that were not vectored $(N=63)$. Figure 6 and 7 show the means of altitude and time, respectively, when departures were cleared to climb to 11,000 feet as a function of gaps at SKYLO and REDDT and Departure Accuracy condition.

Departure Accuracy did have a different impact on aircraft altitude (Figure 6) and time (Figure 7) between gaps at SKYLO and REDDT $(F(3,55)=5.2$, $p<.01$ and $F(3,55)=6.6, p=.001$, respectively). No main effects of gap or departure accuracy were found for altitude or time. However, when gaps were tested separately, Departure Accuracy had a significant main effect on altitude for gaps at $\operatorname{SKYLO}(F(3,23)=$
$4.8, p=.01)$, but not $\operatorname{REDDT}(F(3,32)=1.3, p$ ns $)$. Departure Accuracy also had a significant main effect on time when gaps at situated at SKYLO $(F(3,23)=$ 9.0, $p=.000)$, but not at $\operatorname{REDDT}(F(3,32)=1.0, p$ $\mathrm{ns})$.

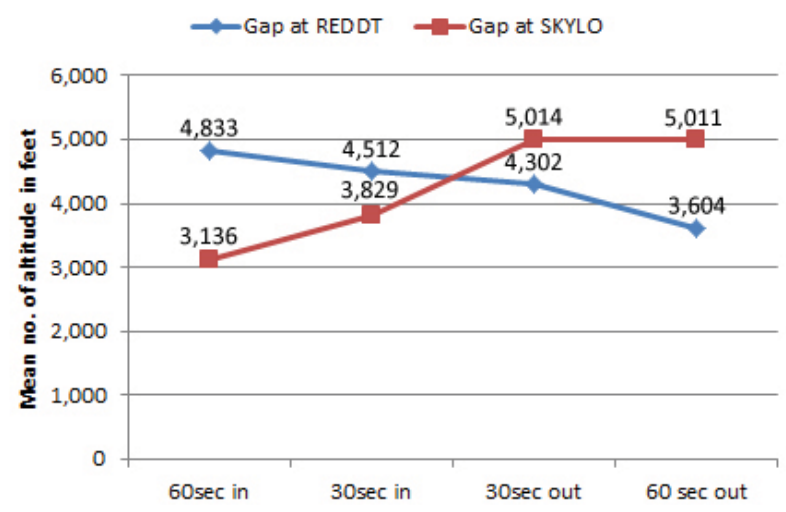

Figure 6. Altitude by Gaps and Accuracy

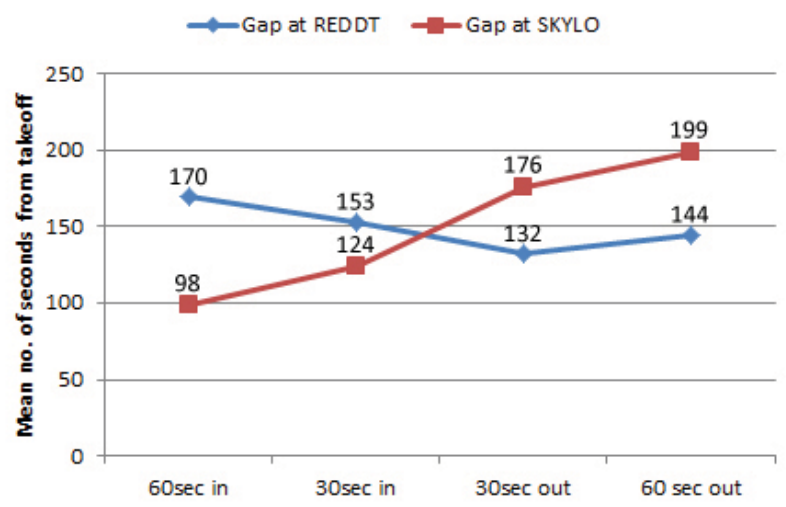

Figure 7. Time by Gaps and Accuracy

When gaps were situated at SKYLO, the more departures were predicted to fly inside the gaps, the earlier departures were cleared and the lower altitude they were. Departures scheduled 60 seconds inside the gaps were cleared at 3,136 feet and 98 seconds after takeoff. Departures scheduled 30 seconds inside the gaps were cleared a little higher $(3,829 \mathrm{ft})$, and approximately 30 seconds later $(124 \mathrm{sec}$ after takeoff). Departures scheduled 30 or 60 seconds outside the gaps were leveled at around 5,000 feet when they were cleared around 3 minutes (176sec \& 199sec respectively) after takeoff.

When gaps were situated at REDDT, time and altitude did not differ significantly. Altitudes seemed to follow an inverse pattern. Departures seemed to be cleared to climb at a lower altitude $(3,604 \mathrm{ft})$ and 
earlier than for aircraft scheduled inside the gaps when departures were scheduled 60 seconds outside the gap. It is possible controllers were trying to climb early to top the arrival traffic at REDDT. Overall departures were cleared to climb before they reached 5,000 feet.

These results show that gaps in the first arrival flow were used opportunistically to climb aircraft above the second arrival flow. But when gaps were scheduled at the first arrival flow, scheduling aircraft inside gaps had a significant impact on the departures' climb profile.

\section{Safety}

Often, controllers opted to climb through gaps at SKYLO and climb departures above the arrivals at REDDT, instead of keeping departures under the Modesto flow and climbing them through gaps at REDDT. They also vectored departures to avoid arrival traffic at REDDT. Were those decisions safe?
Aircraft in the TRACON airspace need at least 1,000 feet vertical separation and 3 nautical miles lateral separation. This rule applies as long as aircraft are on converging trajectories. Once one aircraft has crossed in front or behind of another aircraft, its trajectory is diverging, and the minimum separation requirement no longer applies.

We measured the distance of arrival aircraft relative to the departure aircraft when it crossed the Modesto flow (at or near SKYLO) and also when it crossed the Oakland arrival flow (at or near REDDT) for each type of decision the controller made for the departures as shown in Table 5. This table shows the number of arrival aircraft for a relative distance. Minus numbers are distances of arrivals that have passed in front of the departures, and thus are laterally diverging from departures. The positive numbers are distances of arrivals that are converging. Letters a) through $\mathrm{f}$ ) correspond to the possible states of departures in regards to the Modesto arrivals (a to d) and the Oakland arrivals (e \& f).

Table 5. Lateral Separation

\begin{tabular}{|l|l|l|l|l|l|l|l|l|l|l|l|l|l|}
\hline & \multicolumn{7}{|c|}{ Distance in nm of arrival aircraft to departure aircraft } \\
\hline State of REDDT1 Departure & $\mathbf{-}$ & $\mathbf{1 0}$ & $\mathbf{- 8}$ & $\mathbf{- 6}$ & $\mathbf{- 4}$ & $\mathbf{- 2}$ & $\mathbf{0}$ & $\mathbf{2}$ & $\mathbf{4}$ & $\mathbf{6}$ & $\mathbf{8}$ & $\mathbf{1 0}$ & $\mathbf{1 2 +}$ \\
\hline $\begin{array}{l}\text { Crossing Modesto flow (SKYLO) } \\
\text { a) Route / Level }\end{array}$ & & & & & & & & & & & \\
\hline b) Vectored / Level & & & & 1 & & & 2 & 3 & 2 & 4 & 1 & \\
\hline c) Route / Climbing & & & & & 2 & & & 1 & 1 & 3 & 7 & 22 \\
\hline d) Vectored / Climbing & & & & & & & & 3 & 5 & 3 & 9 \\
\hline$\quad$ Crossing Oakland flow (REDDT) & & & & & & & & & & & & \\
\hline e) Vectored / Climbing & & & & & & & & & 3 & 5 & 3 & 10 \\
\hline f) Climbing (after level under Modesto) & 26 & 2 & 6 & 4 & 2 & 0 & 2 & 3 & 1 & 6 & 8 & 22 \\
\hline
\end{tabular}

In a) departures were leveled and stayed on their leveled route. Eleven arrival aircraft were less than 4 miles away $(0,2)$ from SKYLO. In this case, climbing the departures would have caused a loss of separation. In b) departures were leveled but were vectored to the left of their route at SKYLO. Five arrival aircraft were within $-/+4$ miles of the departures $(-4,-2,0,2,4)$. Two arrival aircraft were less than 3 miles away. As with letter a), departures could not be climbed due to traffic. In c) departures were on their route but were climbed before crossing SKYLO. In this case, no aircraft should be found less than 4 miles of SKYLO. There were 2 arrival aircraft that were within 2 miles but they had past SKYLO, i.e., were diverging. In d) departures were vectored and climbing. No traffic was within 4 miles of the departures. In e) no arrival aircraft was found within 4 miles of the departures. In f) departures had leveled at SKYLO and then were climbed before REDDT. This requires that there be no traffic at REDDT within a $3 \mathrm{~nm}$ lateral distance or that departures are cleared vertically to top arrivals. There were at least 6 arrival aircraft that were within $3 \mathrm{~nm}$ of the departures. The departures flew above the arrivals. However, given the short distance from the Modesto flow and the Oakland flow (4 $\mathrm{nm})$, these departures 
often came close to the minimum lateral separation while being still within $1000 \mathrm{ft}$ of vertical clearance. These 6 departures should have been kept at a level altitude to fly under the arrival flow. In one instance, a departure came close to losing separation.

Overall, controllers were able to tactically vector and climb aircraft. Controllers tried to climb departures early and had to vector them to keep away from arrivals. This was a safe maneuver. However, most of the time, controllers kept departures on their trajectory and cleared them to climb. This decision is safe if aircraft can climb prior to SKYLO. Otherwise, departures could risk losing minimum separation.

\section{Workload and Acceptability}

Controllers responded to an online survey at the end of each run and a post-sim survey at the end of the simulation. Answers were either binary (yes/no), or were scored on a 5-point Likert rating scale, ranging from 1 (lowest) to 5 (highest). Space was made available for comments. The post-run data was analyzed with repeated measures ANOVAs.

\section{Post-Run Survey Results}

No main effect of First Sector and Tool Reliability were found in the survey responses.

Across all runs, the Mulford controller reported a higher mental activity during the busiest time (between "moderate" \& "somewhat high", $M=3.3$, $S D=1.0$ ) compared to the other controllers (between "very low" \& "somewhat low", $M=1.7, S D=.4$ ), $F(1,11)=31.6, p<.001$. Mulford also reported a higher time pressure ("moderate", $M=2.9, S D=0.7$ ) than other sectors (between "very low" \& "somewhat low", $M=1.3, S D=0.2)$ at the busiest time, $F(1,11)$ $=76.5, p<.001$. The Mulford controller also reported being less able to maintain adequate separation ("somewhat high" $M=3.9, S D=1.2$ ) than other controllers ("very high", $M=4.7, S D=0.3 ; F(1,11)$ $=4.7, p=.05$ ).

Although Final and Niles gave a maximum score of 5 , or "Very acceptable," to all questions about the acceptability of allowing the REDDT1 departure through the arrival airspace in regard to workload, coordination and safety in the previous run, REDDT1Mulford's answer was in between "Somewhat acceptable" and "Very acceptable", $M=$ $4(S D=1.1)$ for workload, $M=4.2(S D=0.9)$ for coordination, and $M=4(S D=1.2)$ for safety
$(F(2,22)=9.4$ for workload, 9.2 for coordination, 8.3 for safety, $p<.01$ for all).

One controller stated twice that "Had Mulford been in communication with the REDDT departure in a more timely fashion, it could have been turned and climbed more efficiently." This comment was made after the case of the near loss of separation, when Toga was the first sector to control the departures.

The Mulford controller thought the coordination was cumbersome at times. Mulford used point outs during every run, and engaged in additional verbal coordination during 10/12 runs. Mulford initiated the point out coordination with other sectors, whereas the other sectors only responded to Mulford's requests, sometimes after a delay. The time pressure was therefore on Mulford's shoulders. Mulford reported having time pressure on at least one coordination in each run on $33 \%(4 / 12)$ of the runs; Niles and Final reported none. Mulford indicated that at times he had to wait for other sectors to accept point-outs, qualifying these as "late point-out". Typical comments were "Had to wait on Niles to accept point out when I would have liked to start the aircraft climbing (Run 1) and "Needed to call Niles to get him to accept point out" (Run 11). Although the Mulford controller considered coordination as being accomplished in a timely fashion in 10/12 runs, in the other two runs he did not. Final and Niles sectors accepted point-outs while referencing other aircraft, in $25 \%$ and $20 \%$ of the runs, respectively, which was thought not to be enough by Mulford, as will be seen from the post-simulation survey results.

\section{Post-Simulation Survey Results}

These results confirmed the finding that having "Mulford first" worked better operationally and "in this simulation" was acceptable in terms of workload safety, and coordination than having direct departures go through Toga first. However, the two Mulford controllers thought the SOAR procedure would be only "somewhat acceptable" or less in the field. One gave a rating of 3 or "somewhat acceptable," the other a rating of 2 to the question, "To the best of your knowledge, how acceptable would SOAR operations be in the field (i.e., with SJC Tower and NCT)?" Finally, the Mulford controllers felt that there should have been more referencing of other traffic in the point outs, each stating that over 7 times in the simulation there was other traffic that should 
have been pointed out before a point out was accepted.

\section{Communication Analysis Results}

According to the procedure, a point out assumed that aircraft would stay on its route. Mulford had to call other sectors if additional coordination was needed, such as vectoring the departure. Since Mulford opted to vector many aircraft, controllers came up with their own convention to reduce verbal coordination. During the first 5 runs, Mulford controller called Niles controller each time he intended to vector a departure. Quickly, requests became minimal (Run 4, Mulford to Niles: "Point-out [callsign] about to go northbound with him", Niles: "Approved, [initials]"). In the $6^{\text {th }}$ run, Mulford asked to obtain control for climb and turn for all the Reddt departures:

Niles: "Niles."

Mulford: "Yeah [name] can I have control for these Reddt guys west of the routing and climb as well."

Niles: "Yes you can."

Mulford: "Thank you sir."

Niles: "[initials]."

After that, for each run Mulford would contact Niles and set the same pre-arranged coordination procedure for the remaining departures of that run. Overall, verbal coordination decreased across the runs, from 12 exchanges in the first 4 runs, down to 7 in the second 4 runs, to 6 in the third 4 runs, to 5 in the last 4 runs. Verbal coordination became exceptions to the pre-arranged coordination. Controllers were trying to minimize their coordination effort by establishing rules that do not require verbal repetitions. Such ad-hoc rule-making is a strategy commonly used by people to minimize their effort to understand each other [11].

\section{Discussion}

Arrival and departure airspaces in metroplex environments are usually independent. Routes are segregated and can be suboptimal. Arrival and departures flows could be better integrated with more precise scheduling capabilities. We presented a more optimal departure route from SJC flying across arrival sectors in the San Francisco metroplex. Departures were scheduled at the runway to cross gaps in arrival flows. We manipulated the sector which controlled the departure after takeoff, as well as the schedule accuracy of the departures. Results show that the earlier the arrival controller could coordinate the departure aircraft with other sectors, the earlier the departures could climb to higher altitude and thus climb in a more efficient way. Controllers reported that time pressure was an important factor for the coordination and the control of the departure aircraft. Controllers preferred Mulford, the arrival sector, to control the departure first rather than Toga, the departure sector. An early control of the aircraft gave the arrival sector more time to make a decision and improved climb performance.

Departures were scheduled to cross gaps at REDDT in the second arrival flow (Oakland arrivals) they were crossing. The first flow (Modesto arrivals) was to be crossed below at SKYLO. However controllers crossed both flows opportunistically and took advantage of natural gaps in the first flow to climb aircraft before they had to level off at 5000 feet. For the first 8 runs departures were scheduled to cross gaps in the second arrival flow, and for the last 4 runs, they were scheduled to cross gaps in the first arrival flow. Results showed that during the first 8 runs controllers climbed all the departures before crossing the second flow regardless of the precision of the departures, instead taking advantage of an early climb to top arrivals. They also vectored departures to avoid separation loss. In the last 4 runs the scheduling tool did become relevant to support the controllers' decision to climb aircraft early. When departures were mistimed and were going to miss the predicted gap, controllers kept them at level altitude. When the departures were on time and were going to fly through predicted gap they could be climbed early. The results showed that the more accurate departures were, the earlier aircraft were climbed and the higher their altitude when crossing the second arrival flow.

Controllers overall rated the departure procedure as safe and acceptable within the simulation, but Mulford controllers rated the procedure as only "somewhat acceptable" or less in the field. Time pressure and mental activity was reported as high on occasions. The Mulford controllers, who had the responsibility to climb departures, reported that point outs could take longer than expected. During the second half of the simulation, the Mulford controller 
would ask the Niles controller permission to climb and turn aircraft for all departures at the beginning of each run. This pre-arranged coordination became a procedure for all REDDT1 departures.

Although workload, coordination, and safety were judged by controllers as acceptable in the simulation, it appears that in the field, controllers will need both improved tools and coordination procedures to support SOAR procedures. Decision support tools and pre-arranged coordination procedures are explored in follow-up studies.

\section{References}

[1] Joint Planning and Development Office, June 2012, Integrated Work Plan for the Next Generation Air Transportation System, retrieved from: http://jpe.jpdo.gov/ee/request/home (accessed June 1st, 2012)

[2] Clarke, John-Paul. B., Liling Ren, Evan McClain, David Schleicher, Sebastian Timar, Aditya Saraf, Donald Crisp, Richard Gutterud, Ryan Laroza, Terence Thompson, Carolyn Cross, Taryn Lewis, and Michael D. Madson, 2010, Evaluating Concepts for Metroplex operations, AIAA 2010-9249, Fourth Worth, Proceedings of the AIAA Aviation Technology, Integration, and Operations Conference.

[3] Capozzi, Brian J, Stephen C. Atkins, and Seongim Choi, 2009, Towards optimal routing and scheduling of metroplex operations, AIAA 20097037, Hilton Head, Proceedings of the AIAA Aviation Technology, Integration, and Operations Conference.

[4] Xue, Min, and Shannon Zelinski, 2012, Optimal integration of departures and arrivals in terminal airspace, AIAA 2012-4977, Minneapolis, Proceedings of the AIAA Guidance, Navigation, and Control Conference.

[5] Kupfer, Michael, Todd J. Callantine, Joey Mercer, Lynne Martin, and Everett Palmer, 2010, Controller-Managed Spacing - A Human-inthe-Loop Simulation of Terminal-Area Operations, AIAA-2010-7545, Toronto, Proceedings of the AIAA Guidance, Navigation, and Control Conference.
[6] Doble, Nathan A., John Timmerman, Ted Carniol, Mark Klopfenstein, Midori Tanino, and Ved Sud, 2009, Linking traffic management to the airport surface, Proceedings of the Eight USA/Europe Air Traffic Management Research and Development Seminar.

[7] Capps, Alan, and Shawn A. Engelland, 2011, Characterization of Tactical Departure Scheduling in the National Airspace System, AIAA 2011-6835, Virginia Beach, Proceedings of the AIAA Aviation Technology, Integration, and Operations Conference.

[8] Engelland, Shawn. A., and Alan Capps, 2011, Trajectory-Based Takeoff Time Predictions Applied to Tactical Departure Scheduling: Concept Description, System Design, and Initial Observations, AIAA 2011-6875, Virginia Beach, Proceedings of the AIAA Aviation Technology, Integration, and Operations Conference.

[9] Prevot, Thomas, Nancy M. Smith, and Everett A. Palmer, 2006, The Airspace Operations Laboratory (AOL) at NASA Ames Research Center, AIAA 2006-6112, Keystone, Proceedings of the AIAA Modeling and Simulation Technologies Conference.

[10] Prevot, Thomas, Paul Lee, Todd Callantine, Joey Mercer, Jeffrey Homola, Nancy Smith, and Everett Palmer, 2010, Human-in-the-loop evaluation of NextGen concepts in the Airspace Operations Laboratory, AIAA 2010-7609, Toronto, Proceedings of the AIAA Modeling and Simulation Technologies Conference.

[11] Clark, Herbert H., 1996, Using language, Cambridge: Cambridge University Press.

\section{Acknowledgements}

We would like to recognize the research and development teams in the Airspace Operation Laboratory at NASA Ames. Their prior research and development of MACS has been essential to the success of this research. We also thank the NASA Airspace Systems Project for funding this work.

\section{2nd Digital Avionics Systems Conference October 6-10, 2013}

